\title{
Aenderung des Charakters der Doppelbrechung in Krystalllinsen
}

von

\author{
G. Valentin.
}

Eine in diescm Archiv Bd. IV S. $227-268$ enthaltene Abhandlung beschäftigte sich vorzugsweise mit denjenigen Linsenpräparaten, die nicht bloss das Kreuz oder die Ilyperbeln, sondern auch isochromatische Curven bei linearer Analyse in linear polarisirtem Lichte zcigen. Linsen der Art sind mittlerweile Gegenstände des IIandcls geworden. Optiker, die sich mit der Ilerstellung von Polarisationsobjecten beschäftigen, fertigen sic nach den gegebenen Vorschriften an. Herr Steeg in II o mburg z. B. übersandte mir Proben, die ich mit bestem Gewissen empfehlen kann.

Will man jene überraschenden Bilder erhalten, so muss man die Linsen vollständig trocknen, so dass sie mehr oder minder hart werden. Dic isochronlatischen linge kommen erst damn in ihrem ganzen Farbenrcichthume zum Vorschein. Die Bereitung dicser ansgetrockneten Präparatc hat keine Schwierigkeiten in Fischlinsen. Sie gelingt auch noch so ziemlich in den Reptilien. Man wird dagegen Dutzende von Sïugethierlinsen durch unregelmässiges Springen bei dem letzten Aus. trocknen verlieren, ehe man einige befricdigende Iraipa- 
rate erhält. Unter zwanzig Menschenlinsen führt kaum eine zum Ziele.

Man ungeht diese Gefahren, wenn man auf die isochromatischen Ringe verzichtet und sich mit Kreuz oder Hyperbeln begnügt. Linsen des Menschen und der Säugethiere brauchen dann nur theilweise einzutrocknen, so dass sie grauweiss und halbdurchsichtig werden. Man kann sie in diesem Zustande beliebig schneiden und dinn in Glycerin oder noch besser eingeschmolzen in Canadabalsam aufbewahren. Lnsere Sammlung besitzt solche Präparate, die mehr als drei Jahre alt sind und sich in dieser Zeit nicht im Geringsten verändert haben.

Wir wollen diese Linsen die halbgetrockneten, die anderen dagegen, welche zugleich die isochromatischen Ringe liefern, die vollständig getrockneten nennen. Man findet fast ausnahmslos, dass diese beiden Arten von Linsenpräparaten entgegengesetzte Charaktere der Doppelbrechung darbieten. Die Krsteren wirken wie eine positive und die Letzteren wic eine negative Platte, wenn man das Kreuz und die Ringe mit den gleichen Bildern eimes senkrecht auf die optische Achse geschliffenen Platte cines einaxigen Krystalles vergleicht - - eine Zusammenstellung, die nur bedingter Weise nach den in der oben crwähnten Abhandlung enthaltenen Thatsachen gestattet ist. Der Leser, der sich hiervon unmittelbar überzeugen will, wird am Besten thun, eine solche Platte von Kalkspath oder Honigstein als Repräsentanten eines negativen und eine von unterschwefelsaurem Blei als Typus eines positiven Körpers zur Ifand zu nehmen. Der Quarz, den man oft verwendet, stört meist durch seine circularpolarisirenden Eigenschaften.

Die isochromatischen Ringe der vollstänlig getrockneten Präparate gestatten manche l'rüfungen, die nit ihnen in den halbgetrockncten hinwegfallen. Fis wurden schon früher (a. a. Orte S. 225) angeführt, wie dic 
Verengerung der Ringe nach der Finschaltung einer senkrecht auf die Achse geschnittenen Kalkspathplatte in möglichst vollkommener paralleler Lage und die Art der Quadrantverschiebung der Ringe unter dem Einflusse einer unter +45 Grad oder -45 Grad orientirten Glinmerplatte von $1 / 4$ Wellenlänge Gangunterschied für Gelb die negative Wirkung solcher Linsenpräparate nachweist. Dasselbe bestätigt sich, wenn man das cine Paar von Ringquadranten durch eine Gypsplatte von gleicher Farbung, z. B. eine solche von Roth dritter Ordnung auslöschen lässt.

Gehen wir zu den hier am leichtesten anwendbaren Prüfungsarten, die für beide Gattungen von Linsenpräraten gelten können, ïber, so dient zunächst am Besten cin unter +45 Grad orientirtes Glimmerblättchen von $1 / 4$ Wellenlänge Gangunterschied für Gelb. Das dunkle Kreuz verwandelt sich dann in zwei Punkte. Ihre Verbindungslinie steht in positiven Körpern senkrecht und in negativen parallel der optischen Achsenebene des Glimmers. Man wird sich überzeugen, dass die vollständig getrockneten Linsenpräparate ein Bild letzterer Art, und die halb getrockneten die erstere Richtung der beiden dunklen Punkte Jiefern.

Der gleiche Unterschied wiederholt sich bei der Einschaltung eines Glimmerblättchens, dessen Gangunterschied kein Viertheil Wellenlänge ausmacht. Das Kreuz verwandelt sich dann in zwei Hyperbeln, deren Lage den Charakter der Doppelbrechung angiebt. Ihre reelle Axe, d. h. die, welche nicht durch sie selbst geht, läuft von rechts nach links, oder in einer auf die unter 45 Grad orientirten Axenebene des Glimmers senkrechter Richtung in positiven, und von vorn nach hinten in negativen Körpern. Die halb getrockneten Linsen entsprechen auch bei dieser Probe den ersteren und die vollständig getrockneten den letzteren. 


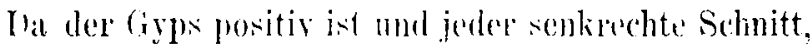
der durch eine anf der optischen Lxe senkrechten Platte seführt wird, cinen Hauptschnitt bildet, so steigt die Farbe cines solehen positiven Krystallblattes in $-4-45$ Grad. wenn die Achenebene des eingeschalteten Gypes unter $+45 \mathrm{Grad}$ eingestelit worden und sinkt in -45 Girad. Liin negativer Körper liefert das lingekehrte. Fin in gleicher Weise orientirter Glimmer hat die entgegengesetzten Wirkungen, weil er selbst zu den negativen lirystallen gehört. Diese doppelte I'robe bestätigt wiederum den erwähnten Unterschied zwischen den beiden Arten von Linsenpräparaten. Sie zcigt zngleich, dass die ganz frische Linse eines eben getüdteten Säugethieres (Kit. ninchens, IIundes), eines lieptiles (Frosch) oder eines Fisches (Forelle) positive Wirkingen hervorbringt, wenn sie selbst noch so durchsichtig ist, dass das dunkle Kreuz bei senkrechtem Durchschnitte der Polarisationsebenen der Nicol noch nicht erkamnt wird. I)er Gebrauch des Gypses ist hier, wie in den meisten anderen Fällen, dem des Glimmers vorzuzichen.

Wir sehen hieraus, dass der positive Jinfluss, welcher der frischen Linse eigen ist, bis zu einem gewinsell Grade des Fintrockneus anhält und später in einen negativen umschlagt. Jlan hat es daher in seiner Gewalt, zwei Jimsenpräparate alls den heiden Augen desselben Thieres mit entgegengesetzten (harakteren der boppelbrechung herzustellen. Der Versuch gelingt leicht an Fischen. Die durch keinc Zerkliiftung gestörte vollständige Austrocknung mit ler negativen Wirkung wird in Säugethierlinsen, die Jahre lang in Weingeist lagen, häufiger, als in frischen erreicht.

Lnsere sitmmlung besitzt zwei in Canalahalsam gefasste Menschenlinsen, die für die Richtigkeit der eben erläuterten Verhältnisse sprechen. Die eine wurle im Freien möglichst vollstindig gutrocknet. Sie zeigt sehr 
vollkommen das Kreuz und einen breiten Ring mit verschiedenen lebhaften Farben an den einzelnen Stellen. Die Prüfung weist ihre negative Wirkung nach. Man liess die zweite in einem verhältnissmässig grossen, hermetisch geschlossenen Raume, bis sie ziemlich hart war, und bearbeitete sie alsdann. Sie giebt kein genügendes Kreuzbild. Die Prüfung mit einem Gypsblättchen zeugt für ihren positiven Einfluss. Halb getrocknete, die Kreuzfigur liefernde Menschenlinsen mit positivem Charakter, die sich Jahre lang in Canadabalsam halten, zuzubereiten, hat keine wesentliche Schwierigkeit.

Diese Beobachtungen bestätigen wiederum die Aehnlichkeit der Linsenpräparate mit den gekühlten oder gepressten Gläsern, die schon in der oben erwähnten $\mathrm{Ab}$ handlung hervorgehoben wurde. Ein Glascylinder, der zuerst vom Umfang aus erwärmt und später mit eincm guten Wärmeleiter umgeben würde, böte in seiner Art einen ähnlichen Uebergang von Positiv zu Negativ dar, wie die Krystalllinsen auf den verschiedenen Stufen der Austrocknung.

Da sich hierbei der Wassergehalt in den verschiedenen Schichten ungleich ändert, so erklärt sich hieraus die Erfahrung von Brewster, der entgegengesetzte Charaktere in den einzelnen Bezirken der Linse hin und wieder angetroffen hat. (Phil. Transact. 1816. p. 312. und Populäre Optik. Uebersetzt von Hartmann. Quedlinburg. 1835. 8. Bd. II. S. 35. 36.) Bliebe es selbst, wie Biot (Troité de Physique expérimentale et mathématique. Tome IV. Paris 1816. p. 574.) annimmt, zweifelhaft, was hier positiv und was negativ war, weil die Lage der optischen nicht vorher bestimmt worden, so berührt dieses doch nicht die Haupterscheinung, dass positive und negative Wirkungen zugleich vorhanden sein können. Diese Jöglichkeit erklärt sich alti don 
Finfliissen ter verschiedenen Grade des Fintrocknens oder ihm ähnlich wirkender Veränderungen.

Sé n ar mon t (Ann. de Chimie et de Physique. 3. Série. Tome XXXIII. 1851. p. 428. 429.) stellte Mischungen des positiven unterschwefelsaueren Bleies und des negativen unterschwefelsaueren Strontianes dar, durch welche dic Doppelbrechung verkleinert, oder selbst für eine grosse Reihe von Strahlen des Spectrums aufgehoben wurde. Andere Combinationen lieferten einen vorherrschend positiven, und noch andere einen negativen Charakter. Man stösst auf Linsen, die auf eincr gewissen Stufe des Eintrocknens ihren entschiedenen Charakter verlieren. Selbst Gypsblättchen geben dann verwirrende Resultate und weisen ungleiche Eigenschaften für verschiedene Stellen nach. Die Doppelbrechung selbst kann dann sehr gering ausfallen.

Die Allotropie der Linsen (a. a. O. S. 242.) kommt bei positivem, wie bei negativem Charakter vor. Sie erinnert einerseits an die Bilder, welche elliptisch polarisirtes Licht liefert, und andererseits in Betreff der Hyperbeln, nicht aber rücksichtlich der isochromatischen Ringe, an die Erscheinungen, die man wahrnimmt, wenn man eine Reihe zweiachsiger Blättchen, z. B. von Glimmer, so übereinander legt, dass sich alle Achsen unter spitzen Winkeln schneiden. Diese gemischten Wirkungen, die eine Folge ungleicher Spannungen bilden und die Linsenpräparate, wie vicle gepresste und gekühlte Gläser zweiachsigen Körpern gleichstellen, können schon durchgreifen, ehe der ursprüngliche positive Charakter verloren gegangen. 